$10-6-2010$

\title{
Auxiliary State Variables for Rotor Crack Detection
}

Zbigniew Kulesza

Bialystok University of Technology, z.kulesza@pb.edu.pl

Jerzy T. Sawicki

Cleveland State University, j.sawicki@csuohio.edu

Follow this and additional works at: https://engagedscholarship.csuohio.edu/enme_facpub

Part of the Mechanical Engineering Commons

How does access to this work benefit you? Let us know!

\section{Original Citation}

Kulesza, Z. and Sawicki, J.T. (2010). Auxiliary State Variables for Rotor Crack Detection. Journal of Vibration and Control, doi: 10.1177/1077546309360050.

This Article is brought to you for free and open access by the Mechanical Engineering Department at EngagedScholarship@CSU. It has been accepted for inclusion in Mechanical Engineering Faculty Publications by an authorized administrator of EngagedScholarship@CSU. For more information, please contact library.es@csuohio.edu. 


\title{
Auxiliary state variables for rotor crack detection
}

\author{
Zbigniew Kulesza' ${ }^{1,2}$ and Jerzy T. Sawicki'
}

\begin{abstract}
In the present study, a new model-based method for rotor crack detection and crack location is proposed. The finiteelement model of the rotor-bearing system accounts for the breathing mechanism of the crack. The model of the rotor system is augmented with an auxiliary single-degree-of-freedom oscillator. The observer is designed and the estimates of its two auxiliary state variables are proposed as crack indicators. The crack location along the shaft is determined by designing a set of observers, which calculate the values of these indicators for different possible crack locations along the shaft. The proposed method is validated numerically and the results prove its capability to detect and locate the crack. Further study will include experimental and numerical investigations to make the approach more robust.
\end{abstract}

\section{Keywords}

Cracks, rotating machinery, observer

\section{Introduction}

Operating rotating machinery with a cracked rotor is very dangerous since the growth of a crack can cause a catastrophic accident if not detected. Transverse cracks in rotors occur due to cyclic loading, creep, stress corrosion, and other mechanisms to which rotating shafts are subjected. An early warning of a crack can considerably extend the durability of these machines, increasing their reliability at the same time.

There are numerous published contributions on the subject of crack modeling, early detection, location and estimation of severity of cracks. Usually, the methods of fault detection and diagnosis for cracked rotors are categorized into three groups: (1) vibration-based methods, which include signal-based (Bently and Muszynska, 1986; Saavedra and Cuitino, 2002) and model-based methods (Bachschmid et al., 2000; Sekhar, 2004; Pennacchi et al., 2006); (2) modal testing methods (Sawicki et al., 2003, 2008, 2009); and (3) nontraditional methods (He et al., 2001; Guo and Peng, 2007; Xiang et al., 2008).

The usual crack detection methods are based on vibration signal analysis. They employ commonly used vibration monitoring equipment (such as proximity probes, phase reference and spectrum analyzers). Steady-state and/or transient vibration data are analyzed for known indicators of cracks. Bently and Muszynska (1986) observed that changes in shaft position and steadily increasing $1 X$ component trends at steady state are reliable crack indicators. Saavedra and Cuitino (2002) presented a theoretical and experimental analysis to demonstrate that the $2 X$ component of vibration for horizontal shafts at half the first critical speed value is a good indicator. Currently, there are two widely industry-accepted approaches for rotor crack detection. One includes monitoring of the changes in amplitude and phase of the synchronous response and the other is based on watching for the occurrence of $2 X$

\footnotetext{
'Center for Rotating Dynamics and Control (RoMaDyC), Fenn College of Engineering, Cleveland State University, Cleveland, OH, USA

${ }^{2}$ Faculty of Mechanical Engineering, Bialystok University of Technology, Bialystok, Poland

Corresponding author:

Jerzy T. Sawicki, Center for Rotating Dynamics and Control (RoMaDyC), Fenn College of Engineering, Cleveland State University, Cleveland, $\mathrm{OH}$, USA

Email: j.sawicki@csuohio.edu
} 
vibrations, especially when the rotor runs near the half of any balance resonance speed.

Nontraditional methods of shaft crack detection include such methods as neural networks, genetic algorithms (He et al., 2001; Xiang et al., 2008), special signal processing techniques, e.g. wavelet (Xiang et al., 2008; Sawicki et al., 2009) and Huang-Hilbert transforms (He et al., 2001), etc. In modal testing methods, changes in system modal characteristics, such as mode shapes and system natural frequencies, response to specially applied excitation (other than unbalance) due to the presence of a crack, are employed for crack detection. Recently, progress in these methods has been made by applying active magnetic actuators to inject a specially designed "diagnostic" force to interrogate the rotating assembly (Ishida and Inoue, 2006; Mani et al., 2006; Sawicki et al., 2008). Many researchers have used a model-based approach for fault detection and diagnosis for cracked rotor systems (Bachschmid et al., 2000; Sekhar, 2004; Pennacchi et al., 2006). Bachschmid et al. (2000) used a least-squares method in the frequency domain to identify and locate machine faults (including a cracked rotor). The crack depth was calculated by comparing the static bending moment due to the rotor weight and the bearing alignment conditions, to the identified "equivalent" periodical bending moment, which simulates the crack. Sekhar (2004) proposed a model-based method that replaced the fault-induced change of the shaft by equivalent loads in the finite-element (FE) model.

One of the techniques applied in model-based methods is state estimation (Patton and Chen, 1997; Isermann, 2005). This approach uses the concept of an observer adopted from classical control theory. Many choices of both linear and nonlinear observers are available and the proper choice depends on the physical properties of the monitored system. Söffker et al. (1993) and Müller et al. (1994) suggested a method of external disturbance reconstruction by using the classical state observer for the extended rotor-bearing system. Simulations have been carried out showing the theoretical success of this method, especially for reconstructing exciting forces as inner forces caused by the crack. Calculating the relative crack compliance as the ratio of additional compliance caused by the crack and undamaged compliance, a clear relation between the opening and closing, and therefore for the existence of the crack, and also about the crack depth is possible.

When noise affects the monitored system, stochastic observers such as a Kalman filter can be used. This is a time domain identification algorithm and Seibold and Weinert (1996) showed that the depth of the crack can be calculated correctly, even if the measurement information is incomplete. The concept of a Kalman filter has been extended to the concept of filter bank in order to detect and diagnose different faults in the vibration system (Loparo et al., 2000). Using state estimation to detect the crack was discussed by Park (2000), who used filter banks with elementary observers to detect the crack occurrence, its depth and location.

The purpose of this study is to develop a new controlbased approach for crack detection by extending and improving the method suggested by Söffker et al. (1993). First, the FE model of the experimental rotorbearing system with a crack is introduced. Then, typical models of crack breathing mechanism are presented, and the model of Mayes and Davies (1984) is chosen for analysis of the considered system. Next, to design a crack-dedicated observer, an auxiliary single-degree-offreedom linear dynamical system is appended to the base system. As a result, two crack indicators as estimates of additional state variables are introduced and the method of crack localization, similar to that suggested by Park (2000), is proposed. Then, the numerical validation of the new method is tested by running several simulations of the considered experimental rotorbearing system. The generated results confirm very good sensitivity of the developed method and its ability to localize the crack along the shaft. In the next step the authors will conduct an experimental study to fully verify predictions of the presented approach.

\section{Rotor-bearing system modeling}

The subject of this study is the crack detection test rig (Figure 1) consisting of a shaft, a disk, and two supporting ball bearings. The notch simulating the crack has been cut using a technique known as wire Electrical Discharge Machining (EDM). The rotor system is also equipped with an active magnetic bearing used as an actuator, injecting specially designed diagnostic force into the system. This force is used in the vibration signal analysis crack detection method, just to compare the results of this method with the results obtained with the method presented here. The details about geometrical and physical properties of the system are given in Section 7.

The rotor-bearing system is modeled using a FE approach. The shaft is discretized into $n$ FEs with six degrees of freedom at each node. At this stage, a shaft element containing the crack will be treated the same as other elements; that is, as an element with no crack. After assembling different shaft elements, adding rigid disks and dynamics of bearings, the complete rotorbearing system is described by the following equations of motion:

$$
M \ddot{x}+\left(D_{d}+\Omega C_{d}\right) \dot{x}+K x=\nLeftarrow F
$$




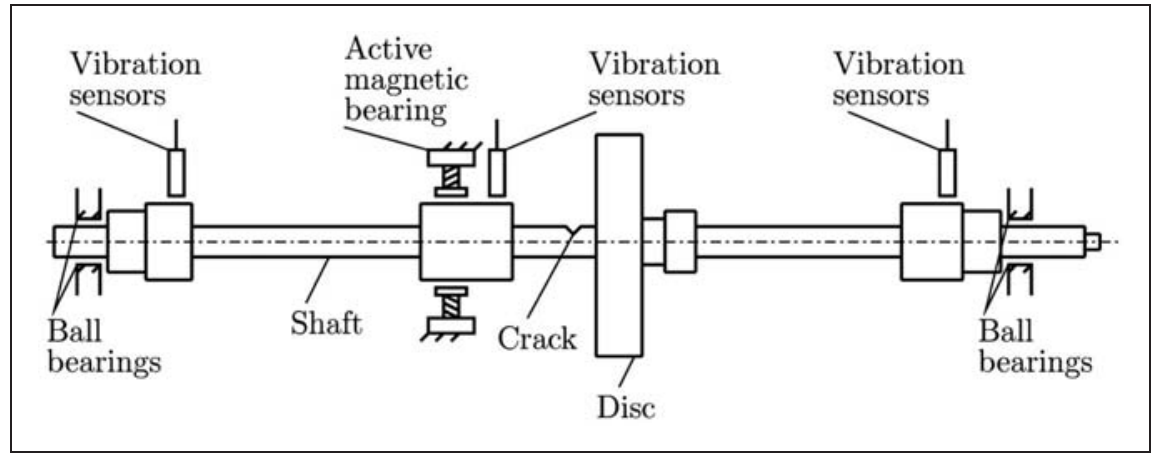

Figure I. Configuration of the rotor-bearing system.

with mass matrix $M$, damping matrix $D_{d}$, gyroscopic matrix $C_{d}$, stiffness matrix $K$, vector of external forces $F$, vector of displacements (linear and rotational) $x$ and rotor spin velocity (Nelson, 1980). Here $M$, $D_{d}, C_{d}, K$ are $6 n \times 6 n$-dimensional matrices, and $F, x$ are $6 n \times 1$-dimensional vectors. For further considerations, we denote $D=\left(D_{d}+\leftarrow C_{d}\right)$.

Applying state space notation, equation 1 can be presented as

$$
\left\{\begin{array}{l}
\left\{\begin{array}{c}
\dot{x} \\
\dot{v}
\end{array}\right\}=A\left\{\begin{array}{l}
x \\
v
\end{array}\right\}+B F \\
=C\left\{\begin{array}{c}
x \\
v
\end{array}\right\}
\end{array}\right.
$$

with $N \times N$-dimensional system matrix $A, N \times N_{i^{-}}$ dimensional control inputs matrix $B$ and $N_{o} \times N$ dimensional measurement outputs matrix $C$. Here, $\left[\begin{array}{cc}x & v\end{array}\right]^{\mathrm{T}}$ denotes the $N$-dimensional state vector, consisting of $6 n$ displacement and $6 n$ velocity variables, that is $N=2 \cdot 6 n$. Here $N_{i}$ denotes number of control inputs and $N_{o}$ number of measurement outputs of the system.Matrices $A, B, C$ are given by

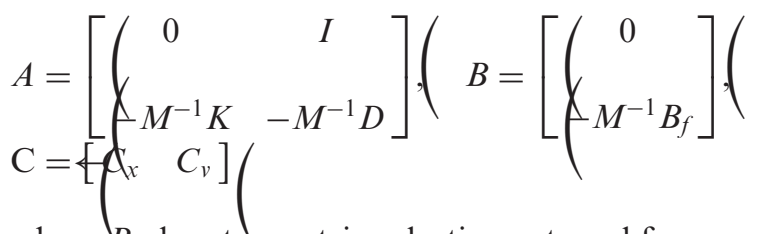

where $B_{f}$ denotes matrix selecting external forces acting on the rotor, and $C_{x}, C_{v}$ matrices representing sensors locations measuring displacements and velocities in selected nodes of the rotor.

Taking into account equation 3, after multiplying and reordering, the first expression of equation 2 can be presented in the following form, which will be helpful in our further considerations

$$
\left\{\begin{array}{c}
\dot{x} \\
\dot{v}
\end{array}\right\}=\left[\begin{array}{c}
v \\
-M^{-1}(K x+D v)
\end{array}\right]+B F
$$

\section{Shaft finite element containing the breathing crack}

In this section, the modeling of the crack and the breathing mechanism are briefly discussed. The first models of the crack accounted for breathing behavior with only two states, i.e. fully opened and fully closed at certain angular position (Gasch, 1993). These models are defined as hinge models. Mayes and Davies (1984) developed a similar model except that the transition from fully open to fully closed is described by a cosine function. Next, Papadopoulos

$(2) \leftarrow$ and Dimarogonas (1987) derived flexibility matrix for the finite shaft element with open crack. Dimarogonas and Paipetis (1983) introduced a full $6 \times 6$ flexibility matrix for a transverse open surface crack on a shaft. Recently, Darpe et al. (2004) provided more detail and complete derivations of the flexibility matrix of a cracked rotor segment starting from Castigliano's theorem. Friswell and Penny (2002) compared several of the simple crack models that may be used for health monitoring, for both linear and nonlinear responses.

In this paper we use the model introduced by Mayes and Davies (1984), who demonstrated that a transverse crack in a shaft can be represented by the reduction of the second moment of area $\Delta J$ of the element at the location of the crack. Using Rayleigh's method, they concluded that the change in $\Delta J$ can be expressed as

$$
\frac{\Delta J / J_{0}}{1-\Delta J / J_{0}}=\frac{R}{l}\left(1-v^{2}\right) F(\mu)
$$

where $J_{0}, R, l, v$, and $\mu$ are the second moment of area, the shaft element radius, the length of the section, the Poisson's ratio, and the nondimensional crack depth, respectively. Here $F(\mu)$ is the fractional change in the second moment of area measured at the crack face. The nondimensional crack depth $\mu$ is given as $\mu=a /(2 R)$, where $a$ defines the crack depth of the shaft. According 
to this, Sinou and Lees (2005) formulated expressions for the reductions of the second moments of inertia

$J_{2}, \quad J_{3}$ about vertical $x_{2}$ and horizontal $x_{3}$ axes of the cracked FE in the rotating coordinates. The stiffness matrix reduction $K_{c}$ for a fully opened crack in rotating coordinates can then be obtained from the flexibility matrix of the uncracked element, as given by Darpe et al. (2004) by substituting reductions $J_{2}$, $J_{3}$ into it. Thus, the element stiffness reduction in orthogonal directions (parallel and perpendicular to the face of the crack) is obtained.

The stiffness matrix of the cracked shaft element can then be presented as (Mayes and Davies, 1984)

$$
K_{c} r=K_{0} \quad f(t) \leftrightarrow K_{c}
$$

where $K_{0}$ is the stiffness matrix of the uncracked shaft element and the so-called crack steering function $f(t)$ is described by

$$
f(t)=\frac{1}{2}(1 \quad \cos t)
$$

where is the angular speed of the rotor. For $f(t)=0$, the crack is totally closed and the cracked rotor stiffness is equal to the uncracked rotor stiffness. For $f(t)=1$ the crack is fully opened.

\section{Auxiliary state variables for crack detection}

Usually, when FE approach is employed, state space variables are displacements and velocities at every node of the discretized model. In fact, the choice of state space variables is not strictly definite and they can be defined in various ways, often without possessing any physical meaning. It should be also noted that the number $N$ of state variables is not definite either.

In this paper we introduce two additional variables, which, as we show later, can be used as very good crack or other rotor-failure indicators.

We assume that after dividing the rotor into FEs, the number of state variables is $N$, and, as usual, they are displacements $x$ and velocities $v$ in subsequent nodes. Aside from this, we assume that there exist two additional state variables $x_{a}, v_{a}$ inside the system, and they can be defined in terms of a single-degree-of-freedom oscillator described as follows:

$$
\left\{\left(\begin{array}{c}
\dot{x}_{a}=v_{a} \\
\dot{a}_{a}=-\frac{k_{a}}{m_{a}} x_{a} \quad \frac{d_{a}}{m_{a}} v_{a}
\end{array}\right.\right.
$$

which can be also presented as

$$
m_{a} \ddot{x}_{a}+d_{a} \dot{x}_{a}+k_{a} x_{a}=0
$$

We assume that $m_{a}, d_{a}, k_{a}$ have such values and that system of equation 9 is stable. Of course variables $x_{a}, v_{a}$ of such a system in steady state will be zero.

Now, equations of motion (equation 1) of the rotorbearing system can be appended by equation 9 yielding the augmented system as follows:

$$
\begin{aligned}
& \begin{array}{ccc}
M & 0 & \ddot{x} \\
0 & m_{a} & \ddot{x}_{a}
\end{array} \quad+\begin{array}{rcc}
D & 0 & \dot{x} \\
0 & d_{a} & \dot{x}_{a}
\end{array} \quad+\begin{array}{rcc}
K & 0 & x \\
0 & k_{a} & x_{a}
\end{array} \\
& =\leftarrow \begin{array}{l}
F \\
0
\end{array}
\end{aligned}
$$

In steady-state behavior, the values of auxiliary $x_{a}$, $\dot{x}_{a}=v_{a}$ remain zero. The auxiliary system is quite independent of the base system and it is impossible to design an observer for the whole combined system due to lack of observability (note zero elements in the matrices).

However, in the case of couplings between auxiliary variables and some state variables of the base system, the system in equation 10 can be expressed as follows:

$$
\begin{aligned}
& \begin{array}{ccc}
M & 0 & \ddot{x} \\
0 & m_{a} & \ddot{x}_{a}
\end{array}+\begin{array}{ccc}
D & D_{a} & \dot{x} \\
0 & d_{a} & \dot{a}_{a}
\end{array}+\begin{array}{ccc}
K & K_{a} & x \\
0 & k_{a} & x_{a}
\end{array} \\
& =\begin{array}{l}
F \\
0
\end{array}
\end{aligned}
$$

or, in a more compact way,

$$
M_{e} \underset{\ddot{x}_{a}}{\ddot{x}}+D_{e} \frac{\dot{x}}{\dot{x}_{a}}+K_{e} \begin{gathered}
x \\
x_{a}
\end{gathered}=F_{e}
$$

where

$$
\begin{aligned}
M_{e}=\underset{M}{\leftarrow} & 0 \\
0 & m_{a}
\end{aligned}, \quad D_{e}=\begin{array}{cc}
D & D_{a} \\
0 & d_{a} \\
K & K_{a} \\
0 & k_{a}
\end{array}, \quad F_{e}=\begin{gathered}
F \\
0
\end{gathered},
$$

While an auxiliary system is independent of the base one (note the zeros in the last row of equation 11), the base system depends on the auxiliary system due to vectors $D_{a}$ and $K_{a}$. These vectors can be seen as additional matrices introducing additional "inputs" $x_{a}$, $\dot{x}_{a}=v_{a}$ into the base system. Again, because the additional system is a stable oscillator with no external forcing, the $x_{a}$ and $\dot{x}_{a}$ will remain zero. Thus, even when 


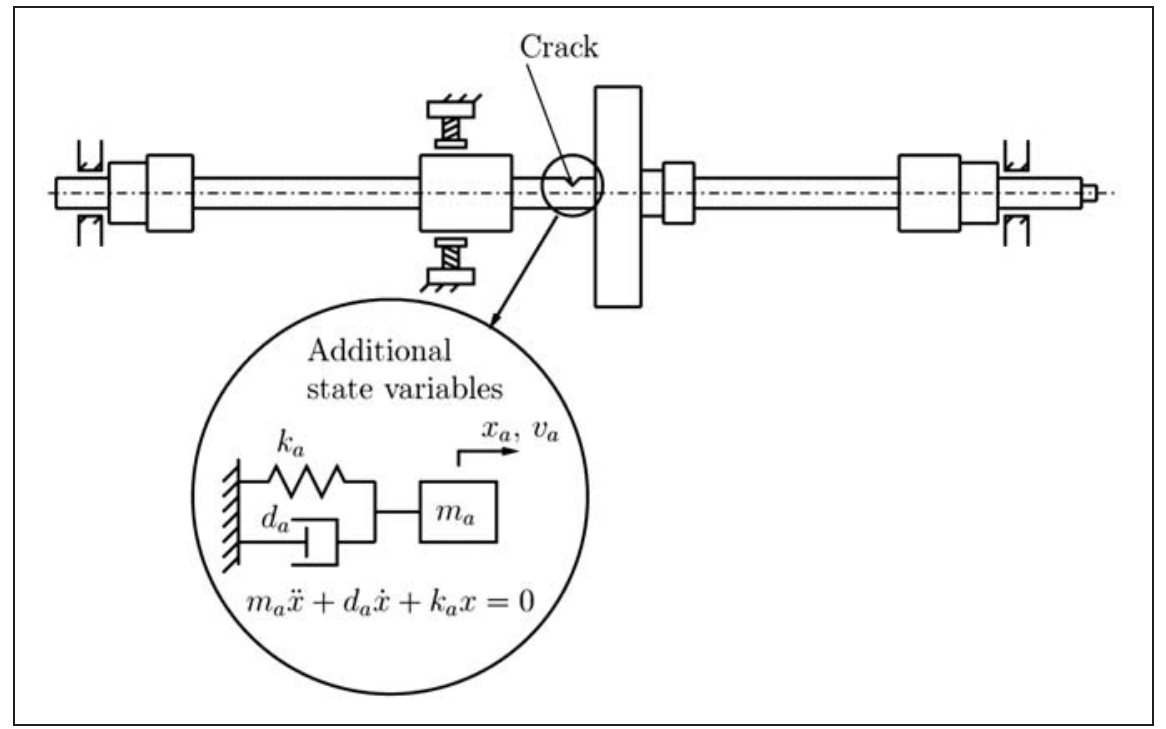

Figure 2. The concept of the crack modeled as two additional states.

couplings $D_{a}$ and $K_{a}$ are possibly present in the given rotor system, there will be no influence of the additional oscillating system on the behavior of the base system. Of course, the given rotor-bearing system can always be seen as having such possible couplings between "real" and some "fictitious" additional states.

With all of the above, let us assume that auxiliary states $x_{a}$ and $v_{a}$ can somehow be changed (for a while, we are not interested in "how"). Of course, through vector $K_{a}$ this would result in the change of stiffness matrix $K_{e}$ (also through $D_{a}$ of damping matrix $D_{e}$ ) of the whole system. It will result in oscillations of the stiffness matrix $K_{e}$. Now, let us recall that the most popular approaches towards modeling the breathing behavior of the crack are based on periodical change of the cracked element's stiffness matrix (Mayes and Davies, 1984; Gasch, 1993; Friswell and Penny, 2002). In this way, the changes of the values of these auxiliary variables can be used to model the crack breathing mechanism or, on the other hand, the crack can be seen as periodical changes of some state variable (or variables) coupled through some vectors $D_{a}, K_{a}$ with the base system (Figure 2).

For the purpose of this paper, we will no longer elaborate on how the additional state variables should change to exactly reflect the breathing mechanism of the cracked shaft, as our main goal is only to develop an accurate method for crack detection. However, we note that even if such auxiliary states have no influence on the base system, they can be observed now (as $D_{a}$ and $K_{a}$ are not zeros). This is certainly true, only if there are a sufficient number of measurements (this means that the pair of matrices $A_{e}, C_{e}$ of the combined system is observable). Thus, we can design an observer for the combined system.

\section{The concept of the new method of crack detection}

In state-space notation, equation 12 can be presented as

$$
\left\{\begin{array}{ccc}
\dot{x}_{e} & & x_{e} \\
\dot{v}_{e} & =A_{e} & v_{e}
\end{array}+B_{e} F\right.
$$

where

$x_{e}=\leftarrow \begin{gathered}x \\ x_{a}\end{gathered}, \quad v_{e}=\leftarrow_{v_{a}}^{v}, \quad A_{e}=\quad \begin{array}{cc}0 & 1 \\ M_{e}{ }^{1} & M_{e}{ }^{1} D_{e}\end{array}$

and

$$
B_{e}=\stackrel{B}{0} 0 \text {, } \quad C_{e}=\left\{\begin{array}{llll}
C_{x} & 0 & C_{v} & 0
\end{array}\right] \quad(16) \leftarrow
$$

Taking into consideration the expressions in equation 13, the combined system matrix $A_{e}$ can be presented as

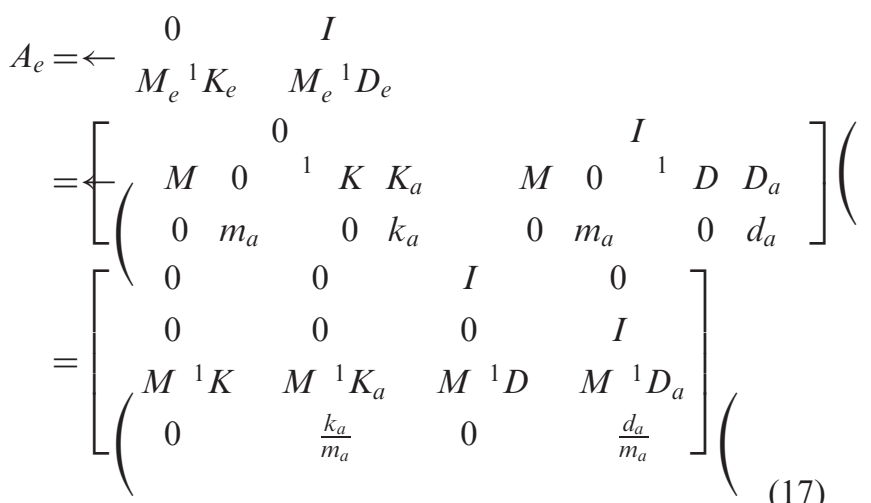


Multiplying and reordering, the first expression of equation 14 can be expressed as the following set of equations:

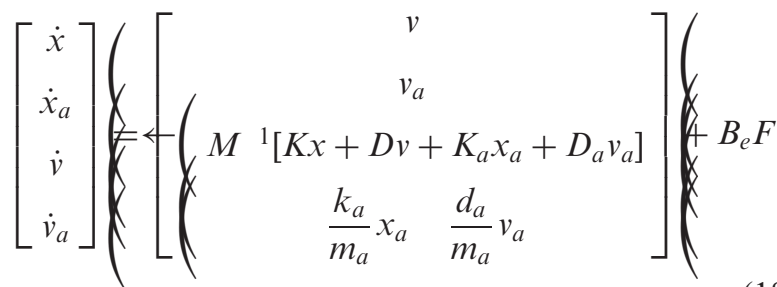

It should be noted that in the case of $x_{a}=0$ and $v_{a}=0$, equation 18 will take the following form:

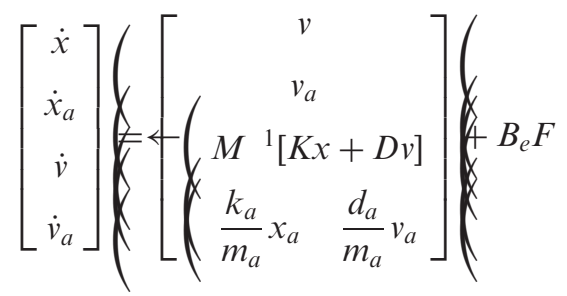

i.e. the first and the third expressions of equation 19 simplify to equation 4 .

Let us assume that the pair $A_{e}$ and $C_{e}$ is observable and let us design an observer for such a combined system. The equations of the observer can be written as (Ogata, 1997)

$$
\left\{\begin{array}{cc}
\left(\begin{array}{c}
\dot{\hat{x}}_{e} \\
\hat{\hat{v}}_{e}
\end{array}=\left(\begin{array}{ll}
A_{e} & L C_{e}
\end{array}\right) \stackrel{\hat{x}_{e}}{\hat{v}_{e}}+B_{e} F+L y\right. \\
y_{o}=I \begin{array}{l}
\hat{x}_{e} \\
\hat{v}_{e}
\end{array}
\end{array}\right.
$$

with $L$ denoting the matrix of observer gains.

Outputs $y_{o}$ of the observer are estimates $\hat{x}_{e}, \hat{v}_{e}$ of the auxiliary states $x_{e}, v_{e}$ of the system. The inputs to the observer are external forces $F$ (unbalance and gravity forces) and measured outputs $y$ of the base rotor system. It should be noted that the same external forces as acting on the real rotor-bearing system is introduced to the observer.

For diagnosis purposes, the output vector $y$ of the rotor system will be measured directly. This vector consists of displacements $x_{r e}$ and/or velocities $v_{r e}$ measured in various points of the real system. It is obvious that in the real system, the values of $x_{r e}$ and $v_{r e}$ can differ from those calculated from equation 4 . This can be written as $x_{r e}=x \pm \delta_{x}$ and $v_{r e}=v \pm \delta_{v}$, where $\delta_{x}, \delta_{v}$ denote deviations of measured displacements $x_{r e}$ and velocities $v_{r e}$ about their theoretical values $x, v$ calculated by equation 4 . These deviations can be caused by a number of mechanisms, but one of them can be possible faults or imperfections in the real rotor-bearing system.

The general concept of crack detection by extended state observer is presented in Figure 3.

After reordering and taking into account the second expression of equation 14, the first expression of equation 20 can be presented as

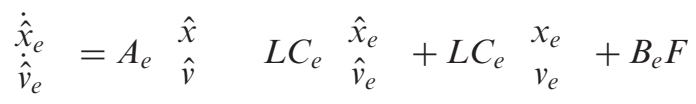

or

$$
\left.\left[\begin{array}{c}
\dot{\hat{x}} \\
\hat{\hat{y}}_{a} \\
\dot{\hat{\hat{v}}}^{\hat{k}_{a}}
\end{array}\right]=A_{e}\left[\begin{array}{c}
\hat{x} \\
\hat{x}_{a} \\
\hat{v} \\
\hat{v}_{a}
\end{array}\right]\right) L C_{e}\left[\begin{array}{c}
\hat{x} \\
\hat{x}_{a} \\
\hat{v} \\
\hat{v}_{a}
\end{array}\right]\left(L C_{e}\left[\begin{array}{c}
x \\
x_{a} \\
y \\
v_{a}
\end{array}\right]\right)\left(B_{e} F\right.
$$

where matrix $L$ of observer gains can be partitioned as

$$
L=\left[\begin{array}{llll}
L_{x} & L_{x a} & L_{v} & L_{v a}
\end{array}\right](\quad(23) \leftarrow
$$

and $\left[\begin{array}{llll}x & x_{a} & v & v_{a}\end{array}\right]^{\mathrm{T}}$ is a vector of states of the rotorbearing system. This vector will be denoted as $u_{1}$, and

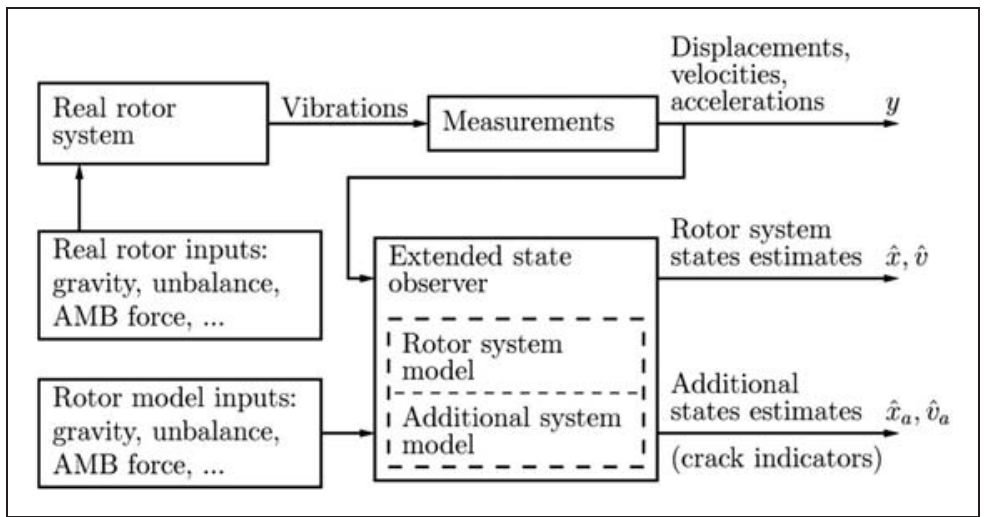

Figure 3. General concept of crack detection by extended state observer. 
because in the steady state $x_{a}=0, v_{a}=0$ (as was stated before), then $u_{1}=\left[\begin{array}{cccc}x & 0 & v & 0\end{array}\right]^{\mathrm{T}}$.

In the ideal case, for the properly designed observer, after a short transient process, its outputs $\hat{x}_{e}, \hat{v}_{e}$ should be exactly the same as the values of states variables $x_{e}$, $v_{e}$ of the system given by equation 12 , i.e. $\hat{x}_{e} \rightarrow x_{e}$, and $\hat{v}_{e} \rightarrow v_{e}$. This is certainly true if its inputs are just known external forces $F$ and exact outputs $y$ (consisting of selected displacements $x$ and velocities $v$ ) of the ideal system (equation 4). In this case, the vector of the observer inputs $u_{1}$ can be written as $u_{1}=\leftarrow$ $\left[\begin{array}{llll}\hat{x} & 0 & \hat{v} & 0\end{array}\right]^{\mathrm{T}}$, and equation 22 will take the following form:

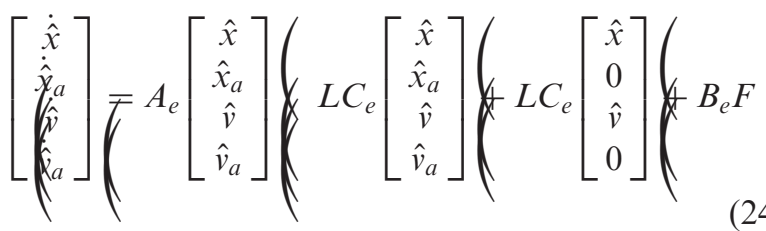

After taking into account equations 16, 17 and 23, equation 24 can be transformed into

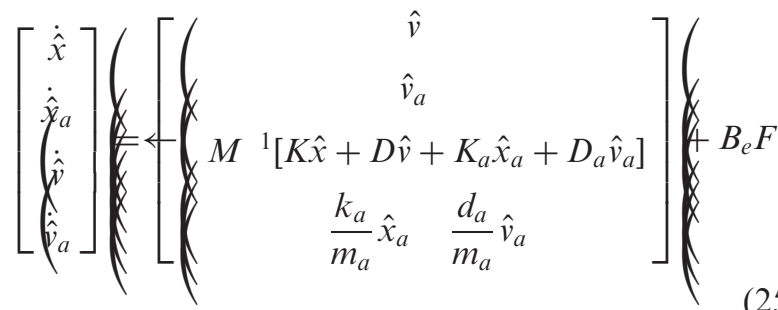

then the vector of the observer inputs $u_{1}$ can be written

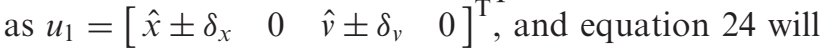
take the following form

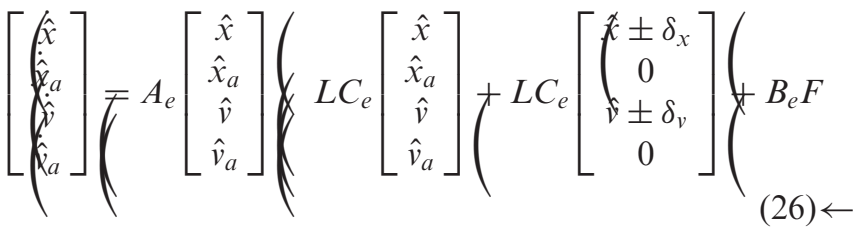

After taking into account equations 16, 17 and 23, equation 26 can be transformed into

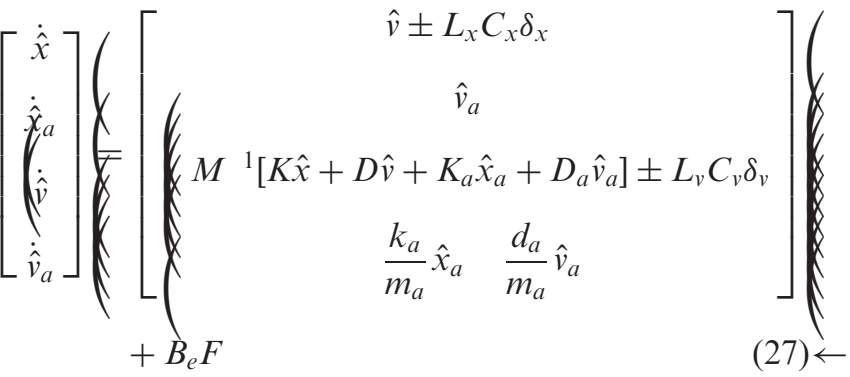

We introduce a new vector $\hat{v}_{1}$, where $\hat{v}_{1}=\hat{v} \pm \leftarrow$ $L_{x} C_{x} \delta_{x}$. Then $\hat{v}=\hat{v}_{a} \pm L_{x} C_{x} \delta_{x}$ and $\tilde{\hat{v}}=\dot{\hat{v}_{1}}$. After introducing this into equation 27 , one will obtain

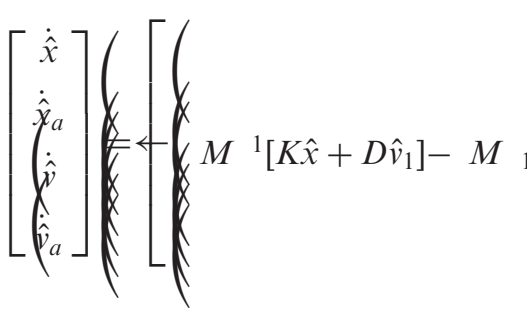

$\hat{v}_{1}$
$\hat{v}_{a}$
${ }_{1}\left[K_{a} \hat{x}_{a}+D_{a} \hat{v}_{a} \pm D L_{x} C_{x} \delta_{x}\right] \pm L_{v} C_{v} \delta_{v}$
$\frac{k_{a}}{m_{a}} \hat{x}_{a} \frac{d_{a}}{m_{a}} \hat{v}_{a}$

From equation 25 one can see that estimates $\hat{x}, \hat{v}$ will tend towards the state variables $x$ and $v$ only if the dynamics of the observer is identical to the rotor-bearing system. This means that equations 25 and 19 should be identical. Obviously, they will be identical only if the last components of the third equation are zeros, which means that the observer must keep $\hat{x}_{a}$ and $\hat{v}_{a}$ equal to zero ensuring that the dynamics of the rotor-bearing system and the observer are the same.

In the case when the inputs to the observer are not equal to ideal $x$ and $v$, but approach the displacements $x_{r e}$ and velocities $v_{r e}$ of the real rotor-bearing system, i.e. $x_{r e}=x \pm \delta_{x}, v_{r}=v \pm \delta_{v}$ or $x_{r e}=\hat{x} \pm \delta_{x}, v_{r}=\hat{v} \pm \delta_{v}$,
It should be noted that this system of equations is identical to equation 19, if the last components of the third equation disappear. Certainly this is possible, only if

$$
M^{1}\left[K_{a} \hat{x}+D_{a} \hat{v}_{a}\right] \pm M{ }^{1} D L_{x} C_{x} \delta_{x} \pm L_{v} C_{v} \delta_{v}=0
$$

or

$$
K_{a} \hat{x}_{a}+D_{a} \hat{v}_{a}= \pm D L_{x} C_{x} \delta_{x} \pm M L_{v} C_{v} \delta_{v} \quad(30) \leftarrow
$$


which means, that the values of $\hat{x}_{a}$ and $\hat{v}_{a}$ cannot remain zero any longer, in order to satisfy the condition expressed by equation 30 .

It is anticipated that the values of $\hat{x}_{a}, \hat{v}_{a}$ will not be stable and can change in time in some way. Thus, absolute mean values $\left|\overrightarrow{\hat{x}}_{a}\right|,\left|\overrightarrow{\hat{v}}_{a}\right|$ along with standard deviations $\sigma\left(\hat{x}_{a}\right), \sigma\left(\hat{v}_{a}\right)$ given by

$$
\begin{aligned}
& \left|\overline{\hat{x}}_{a}\right|=\mid \frac{1}{n_{a}} \sum_{i=1}^{n_{a}}\left(\hat{x}_{a i}|, \quad| \overline{\hat{v}}_{a}|=| \frac{1}{n_{a}} \sum_{i=1}^{n_{a}} \hat{v}_{a i} \mid\right.
\end{aligned}
$$

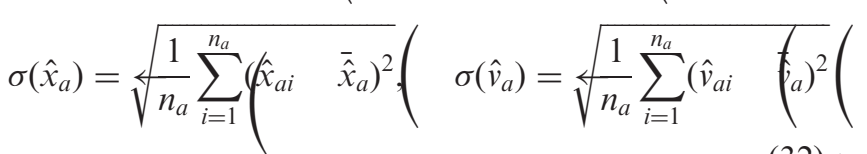

can be used, as more proper crack indicators.

Thus, in the case of a healthy rotor system, its outputs $y$ are proper, i.e. well recognized by the observer. This means that the observer trying to minimize the error between the states of the real system and their estimates will affect only estimates $\hat{x}, \hat{v}$ of states of the base system leaving the estimates $\hat{x}_{a}, \hat{v}_{a}$ of states of the auxiliary system unchanged. As a result, estimates $\hat{x}_{a}$, $\hat{v}_{a}$ should be close to zero.

On the other hand, in the case of a damaged rotor system (e.g. the rotor containing a crack) outputs $y$ are not proper, i.e. they differ from the templates recognized by the observer. This means that this time the observer, by trying to minimize the error between the real states and their estimates, will affect not only estimates $\hat{x}, \hat{v}$ of states of the base system but will be forced to also change estimates $\hat{x}_{a}, \hat{v}_{a}$ of states of the additional system. As a result, estimates $\hat{x}_{a}, \hat{v}_{a}$ will no longer remain zero. Thus, estimates $\hat{x}_{a}, \hat{v}_{a}$ changing their values from zero to some other value can be treated as indicators of possible damage inside the rotorbearing system.

\section{Parameters of the additional system}

To simplify our considerations, and to bring them closer to the real system with a breathing crack, we assume that changes of the auxiliary states can only affect the stiffness of the whole system. Thus, taking $D_{a}=0, K_{a} \neq 0$ transforms equation 30 into

$$
K_{a} \hat{x}_{a}= \pm D L_{x} C_{x} \delta_{x} \pm M L_{v} C_{v} \delta_{v}
$$

As stated above, also the equation 33 shows that the estimate $\hat{x}_{a}$ cannot be zero and can be used as the crack indicator. In order to make the best possible assessment of the existence of the crack, the value of this indicator should be taken as a large as possible nonzero value. It can be seen from equation 33 that for the given mass $M$ and damping $D$ matrices, and deviations/variations $\delta_{x}$, $\delta_{v}$, this can be achieved for low values of the $K_{a}$ vector. However, this is not obvious, as the values $L_{x}, L_{v}$ of the observer gains are also dependent on $K_{a}$, as they are computed based on the system matrix $A_{e}$ (which includes $K_{a}$ ) following the observer design procedure. Thus, it is difficult to determine their rational values by analytical considerations, as gains of the observer are calculated in a numerical way. Consequently, values of $K_{a}$ should also be determined during numerical simulations. The same applies to other parameters of the additional system, namely $m_{a}, d_{a}, k_{a}$. They also should be determined by numerical simulations. To simplify the calculations, we may take $d_{a}=0$. The only important issue concerning values of $m_{a}, k_{a}$ is related to the natural frequency of the auxiliary system, which may affect the behavior of $\hat{x}_{a}, \hat{v}_{a}$ depending on whether the auxiliary system resonates with some frequencies of the base system or not.

An interesting feature of $K_{a}$ vector is that it can be used for estimating the crack localization along the shaft. Let us assume that the crack is located near the middle of the shaft. One should expect, that maximum values $\delta_{x \max }, \delta_{v \max }$ of deviations is located at the middle of vectors $\delta_{x}, \delta_{v}$, i.e.

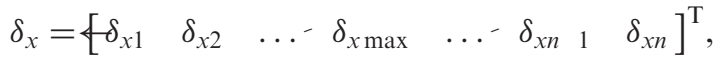

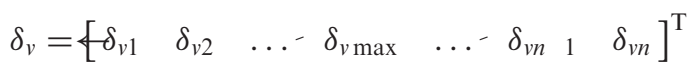

Next, let us assume that we design several observers for the given system, changing only the form of the $K_{a}$ vector. The first observer is designed for $K_{a}$ given as $K_{a 1}=\left[\begin{array}{lllllll}K_{f} & 0 & \ldots & 0 & \ldots & 0 & 0\end{array}\right]^{\mathrm{T}}$, the second for $K_{a 2}=\left[\begin{array}{lllllll}0 & K_{f} & \ldots & 0 & \ldots & 0 & 0\end{array}\right]^{\mathrm{T}}$, the $q$ th for $K_{a q}=\left[\begin{array}{lllllll}0 & 0 & \ldots & K_{f} & \ldots & 0 & 0\end{array}\right]^{\mathrm{T}}$, and the last for $K_{a n}=\left[\begin{array}{lllllll}0 & 0 & \ldots & 0 & \ldots & 0 & K_{f}\end{array}\right]^{\mathrm{T}}$. Then, according to equation 33 , one should expect that the highest values of $\hat{x}_{a}, \hat{v}_{a}$ are obtained for the $q$ th observer. This concept of crack localization is presented in Figure 4.

\section{Numerical investigations}

FE model of the studied rotor-bearing system is shown in Figure 5. The shaft is divided into $n=31$ discrete elements. The 16th element is modeled as the element containing the breathing crack. Its stiffness matrix is expressed in terms of Mayes and Davies (1984) formulae (equation 6). The notch simulating the crack has been cut using EDM with the wire diameter of $1.016 \times 10^{4} \mathrm{~m}$. The model of the system also comprises one disk and two ball bearings. The shaft is $691.4 \times 10^{3} \mathrm{~m}$ in length and $15.875 \times 10^{3} \mathrm{~m}$ in diameter. The disk is $127.0 \times 10^{3} \mathrm{~m}$ 


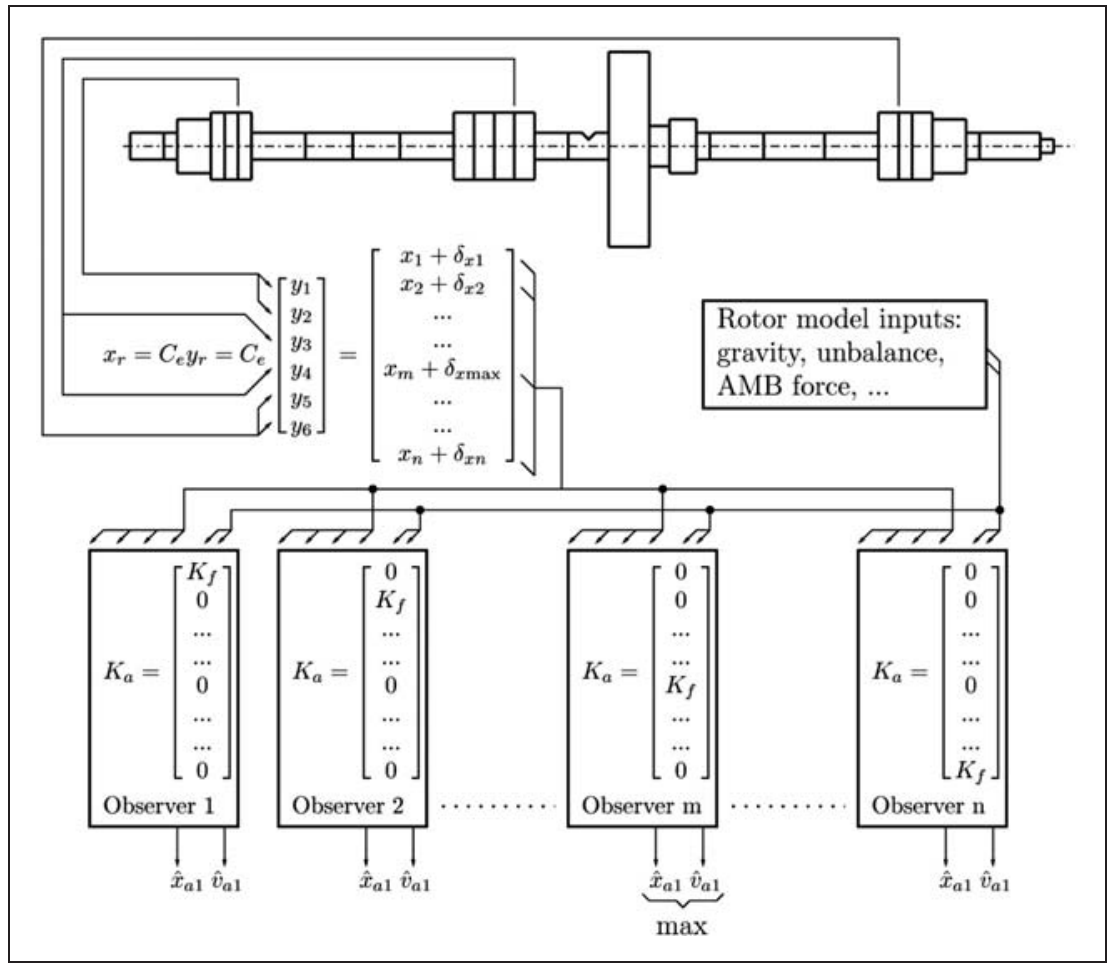

Figure 4. The concept of estimating crack location.

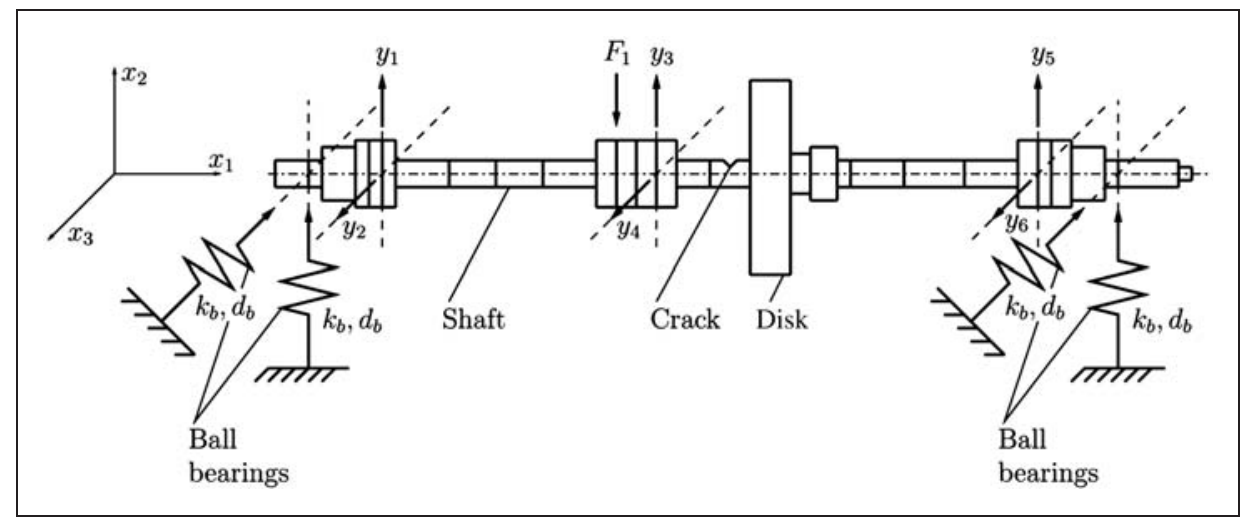

Figure 5. Finite-element model of the rotor-bearing system.

in diameter and $31.0 \times 40^{3} \mathrm{~m}$ thick. The modeled unbalance of the disk is $\varepsilon \notin 4 \times 4 \theta^{3} \mathrm{~m}$. The ball bearings radial stiffness is $k_{b}=3.4 \times 40^{6} \mathrm{~N} / \mathrm{m}$ with damping values assumed to be $d_{b}=40 \mathrm{Ns} / \mathrm{m}$. The rotor is made of steel with modulus of elasticity $E=201.1 \times 4 \theta^{9} \mathrm{~Pa}$, Poisson's ratio $\nu \notin \theta .3$, and density $\rho=7850 \mathrm{~kg} / \mathrm{m}^{3}$. The rotor rotates with the constant angular frequency $=27 \mathrm{~Hz}(1620 \mathrm{rpm})$.

The inputs to the system are gravity and unbalance forces, as well as additional force $F_{a}$, that can be injected by magnetic actuator at the location of the node number 12, as shown in Figure 5. It has been determined that the amplitude of this force is about
$F_{a}=40 \mathrm{~N}$. The outputs of the system are four displacements in vertical and horizontal directions measured near ball bearings, in places denoted as $y_{1}, y_{2}, y_{5}, y_{6}$ and two displacements $y_{3}, y_{4}$ measured near the active magnetic actuator.

The FE model of the base rotor-bearing system consists of $N=384$ state variables, representing 192 nodal displacements and 192 nodal velocities. Time simulations are carried out in an inertial coordinate system, using the Newmark integration method (Xie, 1996). In the case of such a large number of states this method is significantly faster than other ordinary differential equation (ODE) solvers. 


\section{I. Model reduction}

For the purpose of observer design, the model reduction has to be employed. The other advantage of model reduction is minimizing the order of the observer itself, which is an important factor if a hardware implementation of the algorithm is considered.

Equations of motion (equation 1) are reduced using the lower mode shapes of the rotor-bearing system (Preumont, 2008). The reduction procedure is to calculate the eigenvectors of the system as

$$
\left(-\omega_{0 i} M+K\right) \phi_{i}=0
$$

where $\omega_{\mathrm{O} i}$ and $\phi_{i}$ are the $i$ th natural frequency and mode shape. If lower $r$ modes are retained then the reduction transformation is

$$
T_{r}=\left[\begin{array}{lll}
\phi_{1} & \phi_{2} & \ldots-\phi_{r}
\end{array}\right]
$$

The reduced equations of motion are then, assuming proportional damping,

$$
M_{r} \ddot{x}_{r}+\left(D_{r}+\omega C_{r}\right) \dot{x}_{r}+K_{r} x_{r}=F_{r}
$$

where

$$
\begin{aligned}
& x_{r}=T_{r} x, \quad M_{r}=T_{r}^{\mathrm{T}} M T_{r}, \quad D_{r}=T_{r}^{\mathrm{T}} D T_{r}, \\
& C_{r}=T_{r}^{\mathrm{T}} C T_{r}, \quad K_{r}=T_{r}^{\mathrm{T}} K T_{r}, \quad F_{r}=T_{r}^{\mathrm{T}} F
\end{aligned}
$$

We investigated a reduced model for $r=6$. The dynamic properties of the model can be assessed by comparing frequency responses of the reduced and full model. Such comparison is presented in Figure 6.

\subsection{Observer design}

The observer gain matrix $L$ can be calculated using several methods (Ogata, 1997). In this study, the pole placement method and linear-quadratic method using the Riccati equation have been used. In the pole placement method, eigenvalues $\Lambda_{o}$ of the observer matrix $A_{o}=\left(\begin{array}{ll}A_{e r} & L C_{e r}\end{array}\right)$ are chosen such that

$$
\Lambda_{o}=-4 \cdot \ll \operatorname{Re}\left(\Lambda_{e r}\right)+j \cdot \operatorname{Im}\left(\Lambda_{e r}\right) \quad(39) \leftarrow
$$

where $\Lambda_{e r}$ is vector of eigenvalues of reduced combined system matrix $A_{e r}$, and

$A_{e r}=\leftarrow$

In linear-quadratic method, the observer gains $L$ are found by solving the following Riccati equation:

$$
\begin{aligned}
& A_{e r} L E+E^{\mathrm{T}} L A_{e r}^{\mathrm{T}} \quad\left(E^{\mathrm{T}} L C_{e r}^{\mathrm{T}}+S\right) R{ }^{1}\left(C_{e r} L E+S^{\mathrm{T}}\right) \leftarrow \\
& \quad+Q=0
\end{aligned}
$$

where $Q, R, S, E$ are weighting matrices. They are chosen as follows

$$
\begin{aligned}
& \left.Q=\left[\begin{array}{cccc}
q_{1} I_{r \times r} & 0_{r \times 1} & 0_{r \times r} & 0_{r \times 1} \\
0_{1 \times r} & q_{2} & 0_{1 \times r} & 0 \\
0_{r \times r} & 0_{r \times 1} & q_{3} I_{r \times r} & 0_{r \times 1} \\
0_{1 \times r} & 0 & 0_{1 \times r} & q_{4}
\end{array}\right]\right\} \\
& R=r_{1} I, \quad S=0, \quad E=I
\end{aligned}
$$

where $q_{1}=10^{8}, q_{2}=10^{8}, q_{3}=1, q_{4}=1, r_{1}=0.1$.

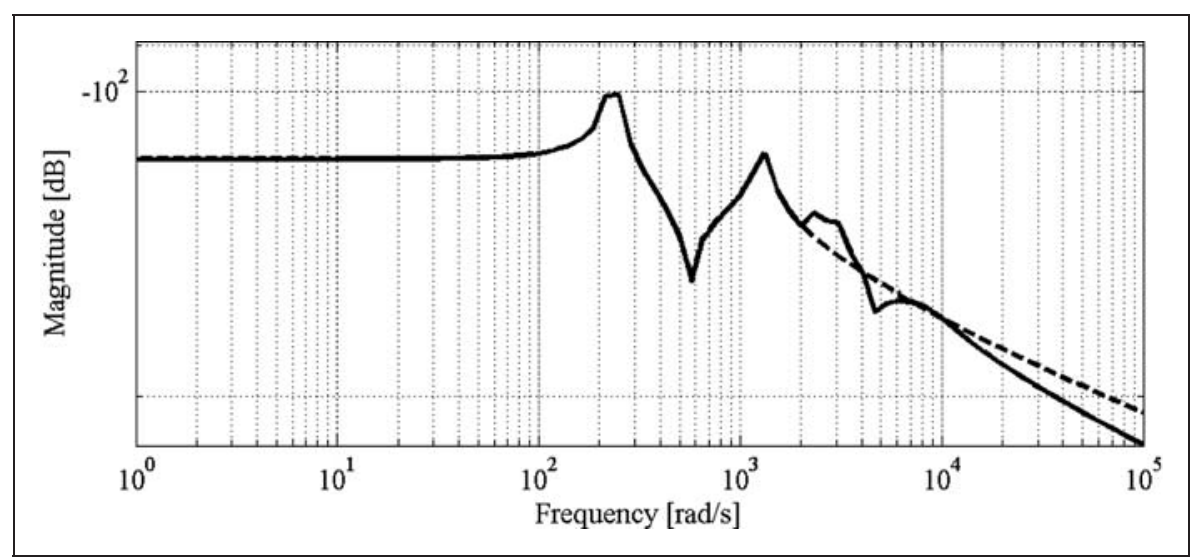

Figure 6. Frequency responses of the reduced and full model for $r=6$. 


\section{Numerical results}

Several preliminary numerical experiments were conducted to determine the parameters of the auxiliary system and the observer, and to test the proposed crack detection method.

First of all, the dynamic properties of the system were determined. The Campbell diagram as well as frequency responses were calculated. The Campbell diagram of the rotor-bearing system is shown in Figure 7. The first two critical bending frequencies are located at $\omega_{c 1}=37.05 \mathrm{~Hz}(2223 \mathrm{rpm}), \omega_{c 2}=229.05 \mathrm{~Hz}$.

Next, the calculations were conducted in the following order:

1. Integration of the equations of motion of the full $\mathrm{FE}$ model of the rotor-bearing system with or without the crack. The input data are full system matrices $A$, $B, C$, gravity, unbalance and magnetic actuator force. The equations are integrated with the Newmark method until a steady state is reached. The results are six displacements $y_{1}, \ldots, y_{6}$ in three selected nodes; the fast Fourier transform (FFT) of the displacements is calculated.

2. Design of the observer for the combined system with the use of one of the methods given in Section 7.2. The input data are the reduced combined system matrices $A_{e r}, C_{e r}$, parameters $m_{a}, k_{a}$ of the additional system, and reduced coupling vector $K_{a r}$. The result is the observer gain matrix $L$.

3. Time simulations of the observer for the reduced combined system. The input data are gravity, unbalance and magnetic actuator force, displacements $y_{1}, \ldots, y_{6}$, reduced combined system matrices $A_{e r}$, $B_{e r}, C_{e r}$, and observer gain matrix $L$. The results are crack indicators: estimates $\hat{x}_{a}, \hat{v}_{a}$ of the

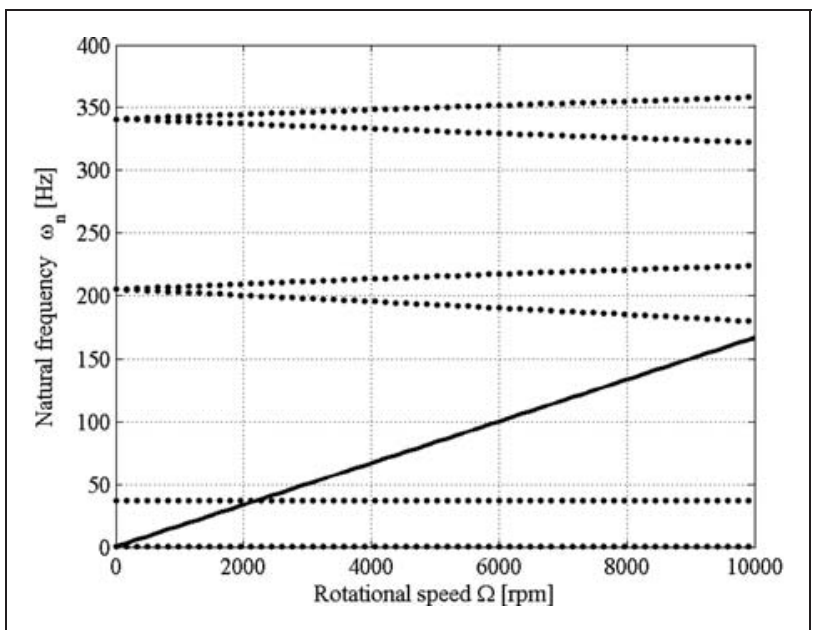

Figure 7. Campbell diagram of the rotor-bearing system. additional states, their absolute mean values $\hat{x}_{a}$, $\hat{v}_{a}$ together with standard deviations $\sigma\left(\hat{x}_{a}\right), \sigma\left(\hat{v}_{a}\right)$.

These calculations were repeated several times for various parameters of the auxiliary system. The observer was calculated using the Riccati equation. Here, vector $K_{a}$ consists of an all-zeros column vector of size $6 n \times 4$, where $n$ number of finite elements, except the $q$ th node element, which is a $6 \times 4$-column vector $K_{f}$ of not-all-zero elements, that is,

$$
K_{a}=\left[\begin{array}{lllll}
0 & 0 & \ldots-K_{f} & \ldots-0
\end{array}\right]^{\mathrm{T}} \quad(43) \leftarrow
$$

where

$$
K_{f}=\left\{\begin{array}{llllll}
0 & k_{f y} & k_{f x} & 0 & 0 & 0
\end{array}\right]^{\mathrm{T}}
$$

and $k_{f x} \neq \theta, k_{f y} \neq 0$. The index $q$ equals 16 for the cracked element, and $1, \ldots, 15,17, \ldots, 31$ otherwise.

Figures $8-13$ present crack indicators $\hat{x}_{a}, \hat{v}_{a}$ for different parameters $m_{a}, k_{a}$ of the additional system and different parameters of the coupling vector $K_{a}$. Parameters $m_{a}, k_{a}$ were chosen in such a way that natural frequencies of the auxiliary system are $\omega_{a n 1}=\leftarrow$ $0.16 \mathrm{~Hz}$ (for $m_{a}=4, k_{a}=4$ ) and $\omega_{a n 2}=37.05 \mathrm{~Hz}$ (for $\left.m_{a}=4, k_{a}=5.12 \times 40^{4}\right)$. As can be seen, the natural frequency $\omega_{a n 2}$ is just the same as the first critical bending frequency $\omega_{c 1}$ of the whole rotor-bearing system.

It can be observed that in almost every case crack indicators $\hat{x}_{a}, \hat{v}_{a}$ generate a very clear evidence of crack existence. Their values for the system containing the crack are far away from zero and they are significantly higher as compared with very low values obtained for the system without the crack. In particular, for low values of the vector $K_{a}$ and natural frequency $\omega_{a n 1}=0.16 \mathrm{~Hz}$, they indicate the crack very well. What is more important, for $\omega_{\text {an } 2}=37.05 \mathrm{~Hz}$ the absolute mean values $\hat{x}_{a}$ and $\hat{v}_{a}$ change, as the index $q$ of the possible cracked element changes within the $K_{a}$ vector. It can be clearly seen when comparing Figures 11, 12 and 13. This means that according to the concept presented in Section 6 it is possible to localize the crack along the shaft length. To study this possibility further, we designed the series of observers for different vectors $K_{a}$, as presented in Figure 4, and calculated mean values and standard deviations of crack indicators for each case. The results are presented in Figures 14 and 15. As can be seen, the higher mean values as well as the standard deviations of crack indicators are located in the area of the shaft element containing the crack. From Figure 14 one can deduce that the crack is located in the 17 th shaft element, as the values of the mean value and standard deviation of $\hat{x}_{a}$ are the highest here. This is very close to the real situation, as the crack is located in the 16th element. 

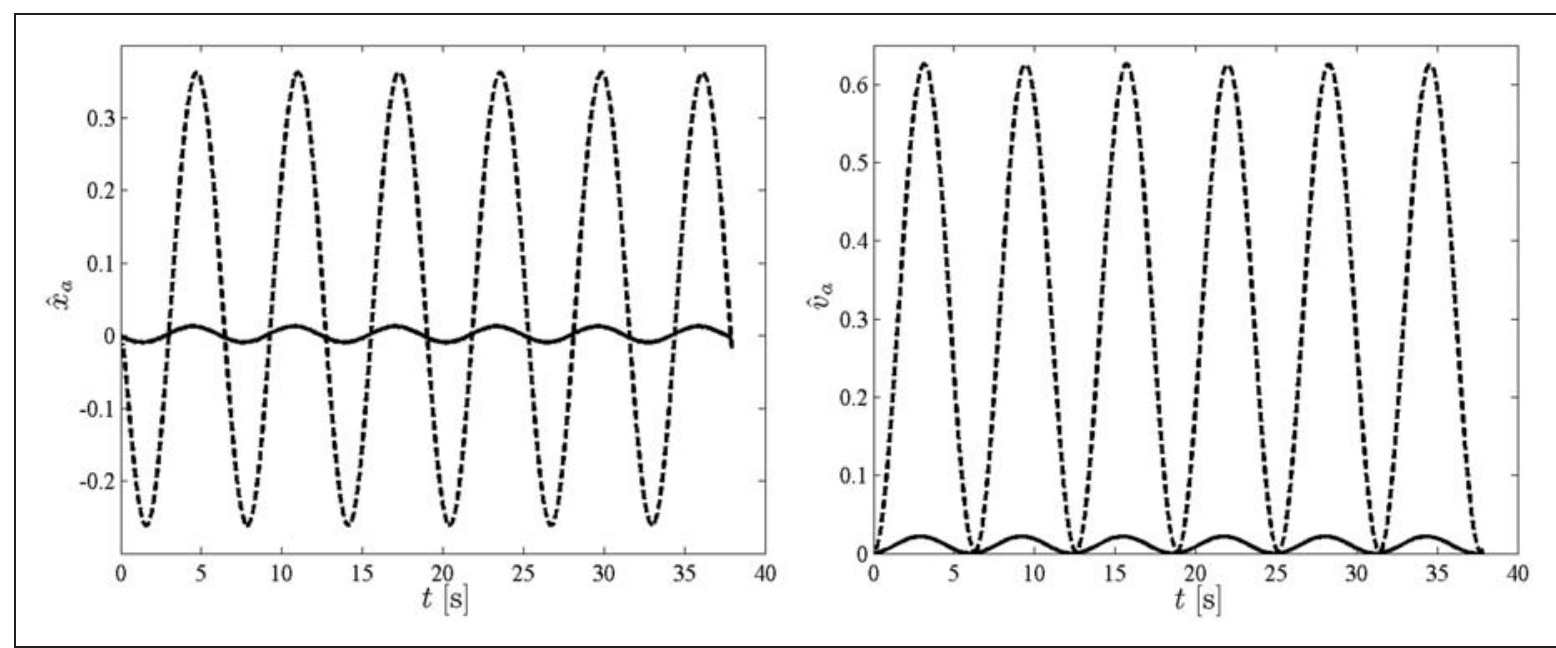

Figure 8. Crack indicators $\hat{x}_{a}, \hat{v}_{a}$ : solid line, no crack; dashed line, $40 \%$ crack; $m_{a}=1, k_{a}=1, k_{f y}=40^{3}, k_{f x}=10^{3}, q=46$.
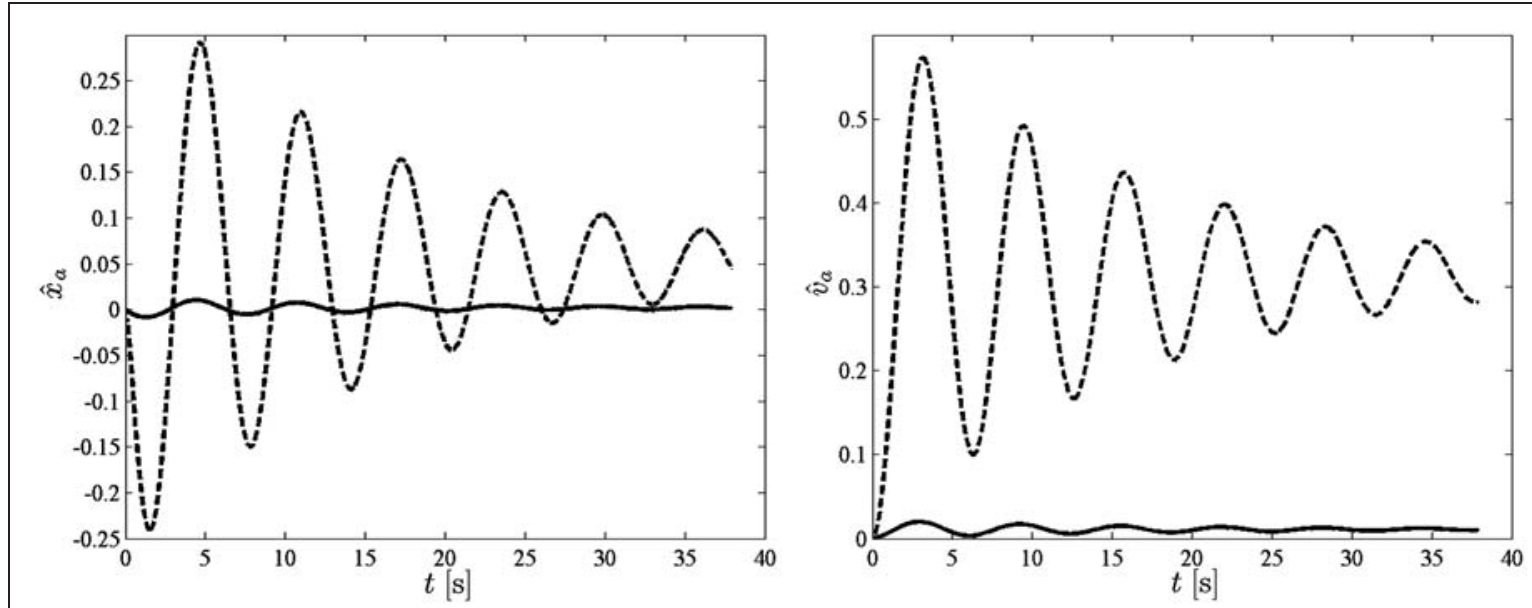

Figure 9. Crack indicators $\hat{x}_{a}, \hat{v}_{a}$ : solid line, no crack; dashed line, $40 \%$ crack; $m_{a}=I, k_{a}=I, k_{f y}=4, k_{f x}=I, q=46$.

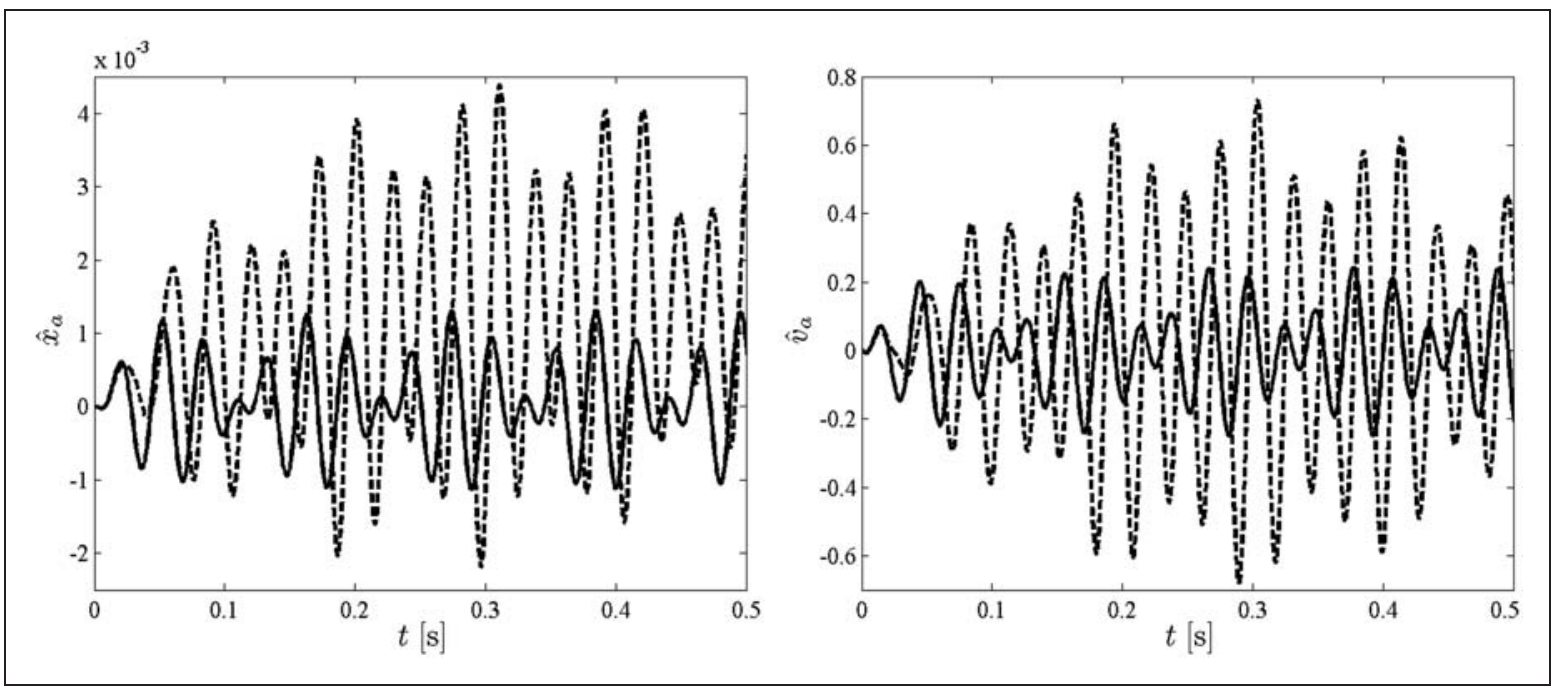

Figure 10. Crack indicators $\hat{x}_{a}, \hat{v}_{a}$ : solid line, no crack; dashed line, $40 \%$ crack; $m_{a}=4, k_{a}=5.12 \times 40^{4}, k_{f y}=10^{3}, k_{f x}=40^{3}$, $q=46$. 


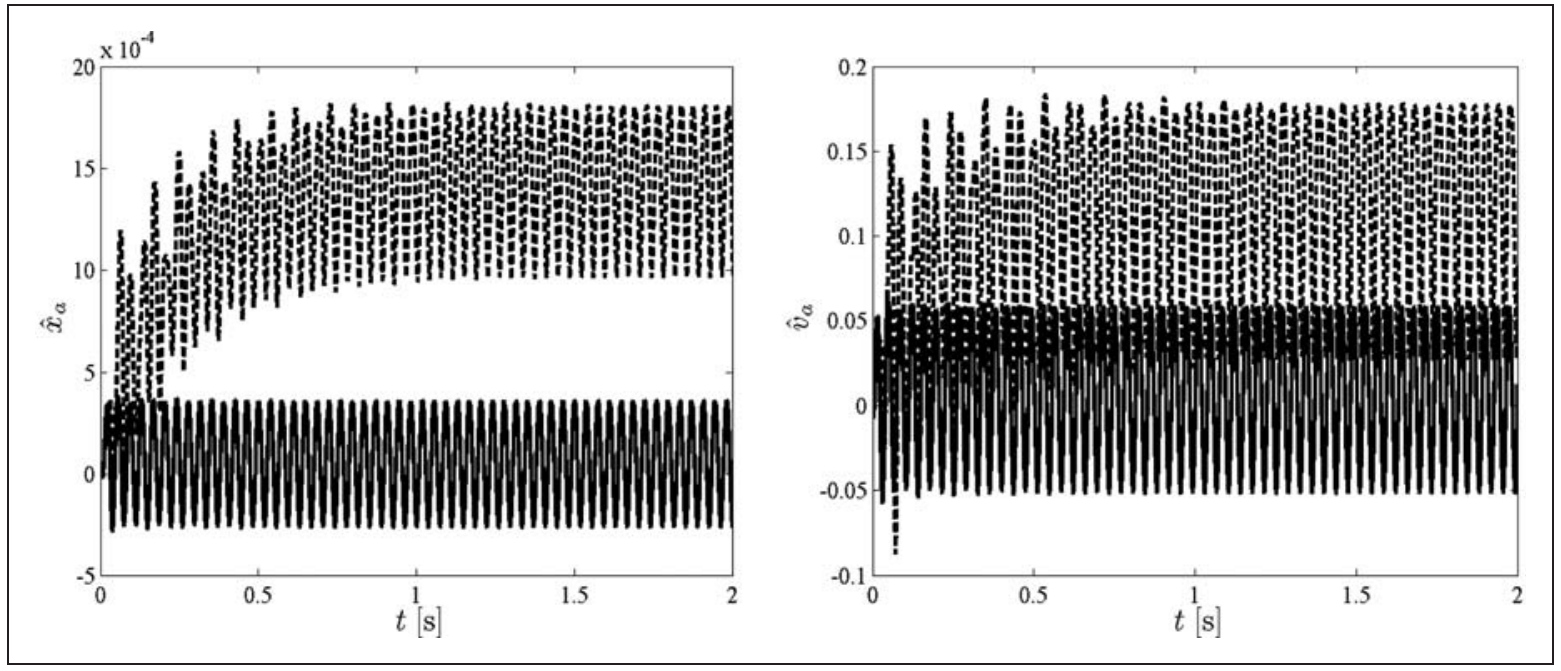

Figure II. Crack indicators $\hat{x}_{a}, \hat{v}_{a}$ : solid line, no crack; dashed line, $40 \%$ crack; $m_{a}=4, k_{a}=5.12 \times 40^{4}, k_{f y}=4, k_{f x}=1, q=46$.

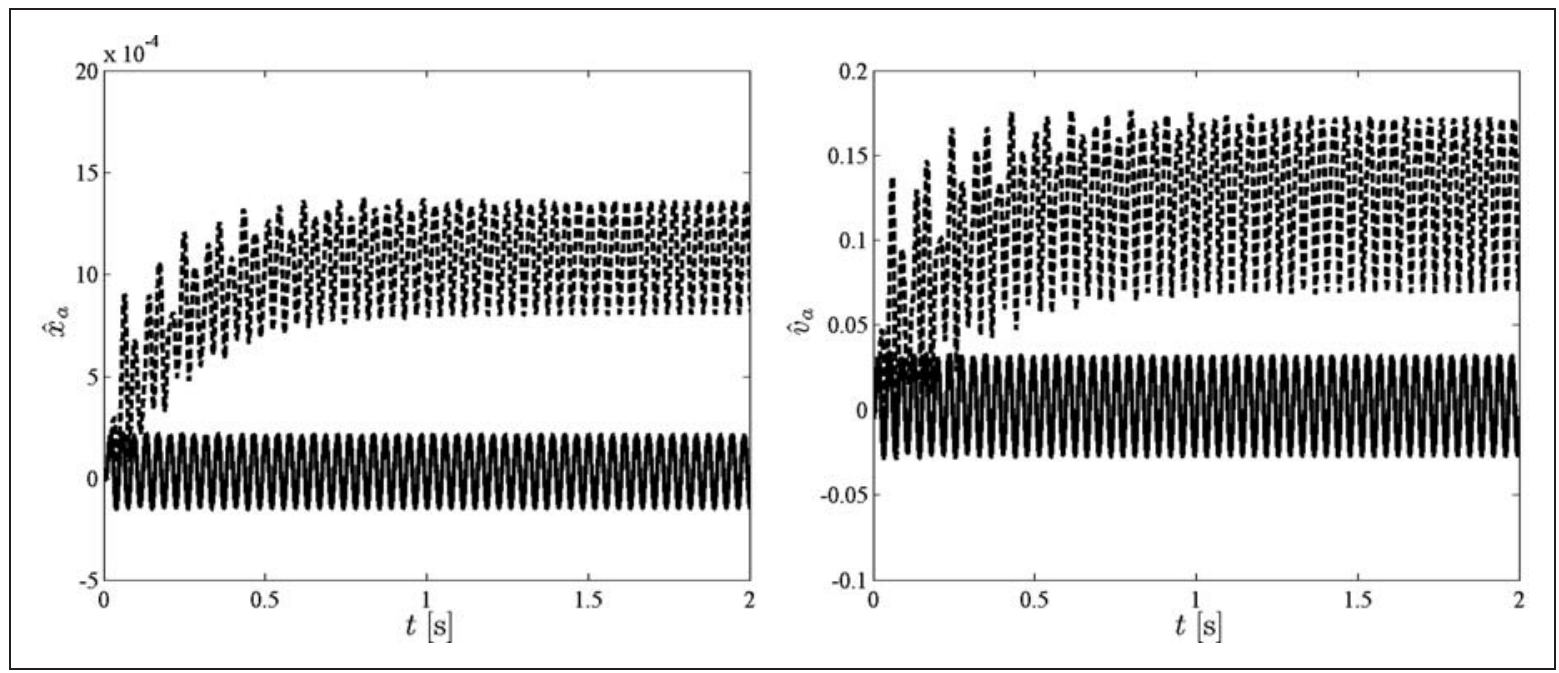

Figure 12. Crack indicators $\hat{x}_{a}, \hat{v}_{a}$; solid line, no crack; dashed line, $40 \%$ crack; $m_{a}=4, k_{a}=5.12 \times 40^{4}, k_{f y}=4, k_{f x}=1, q=42$.

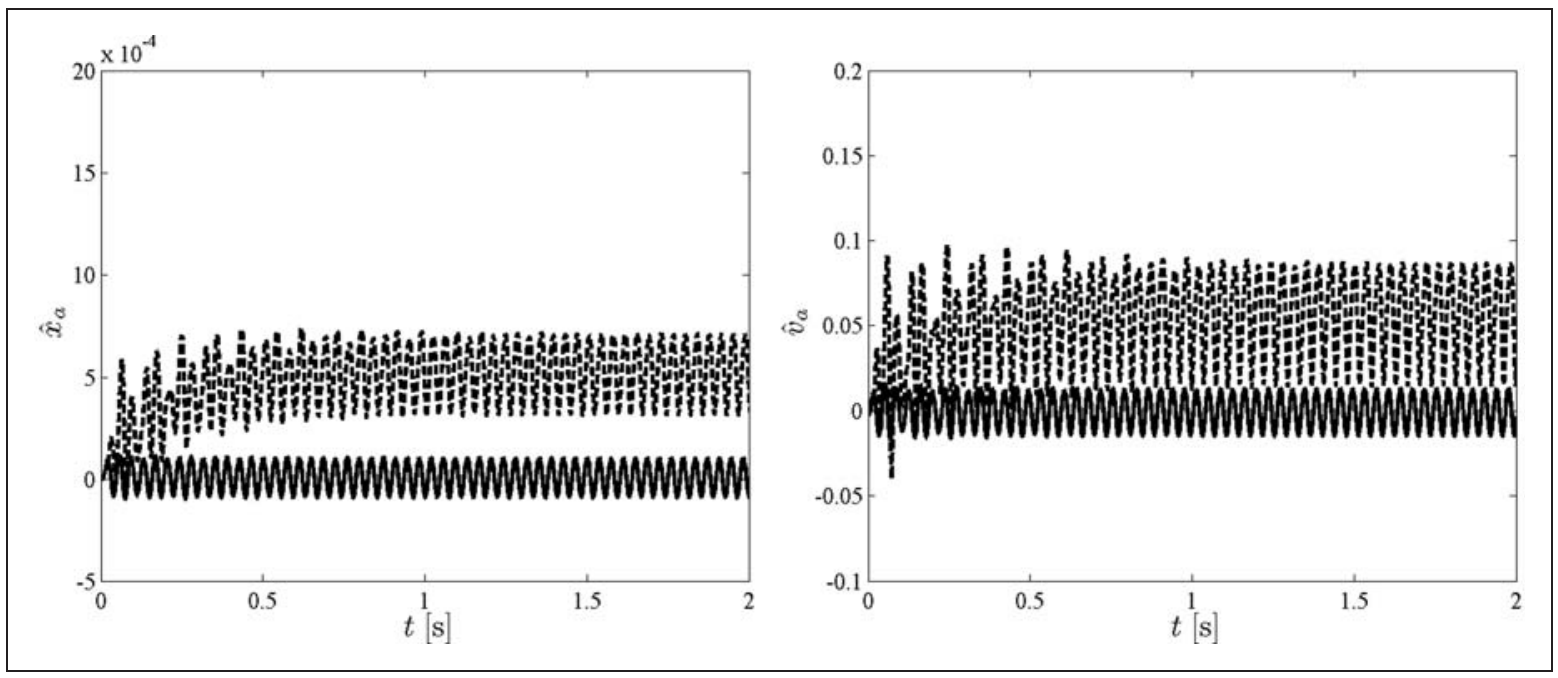

Figure 13. Crack indicators $\hat{x}_{a}, \hat{v}_{a}$; solid line, no crack; dashed line; $40 \%$ crack; $m_{a}=4, k_{a}=5.12 \times 40^{4}, k_{f y}=4, k_{f x}=4, q=5$. 


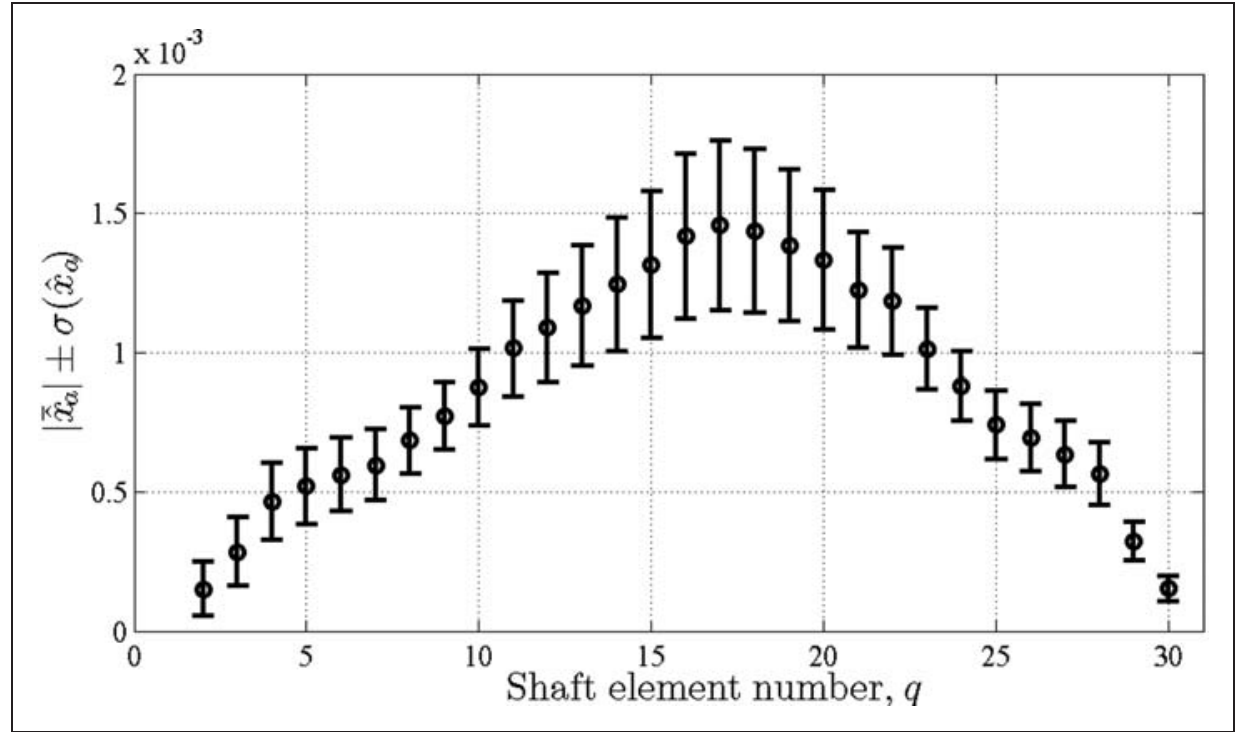

Figure 14. Absolute mean values $\hat{x}_{a}$ and standard deviations $\sigma\left(\hat{x}_{a}\right)$ fer different locations $q$ of vector $K_{f}$ within vector $K_{a}$.

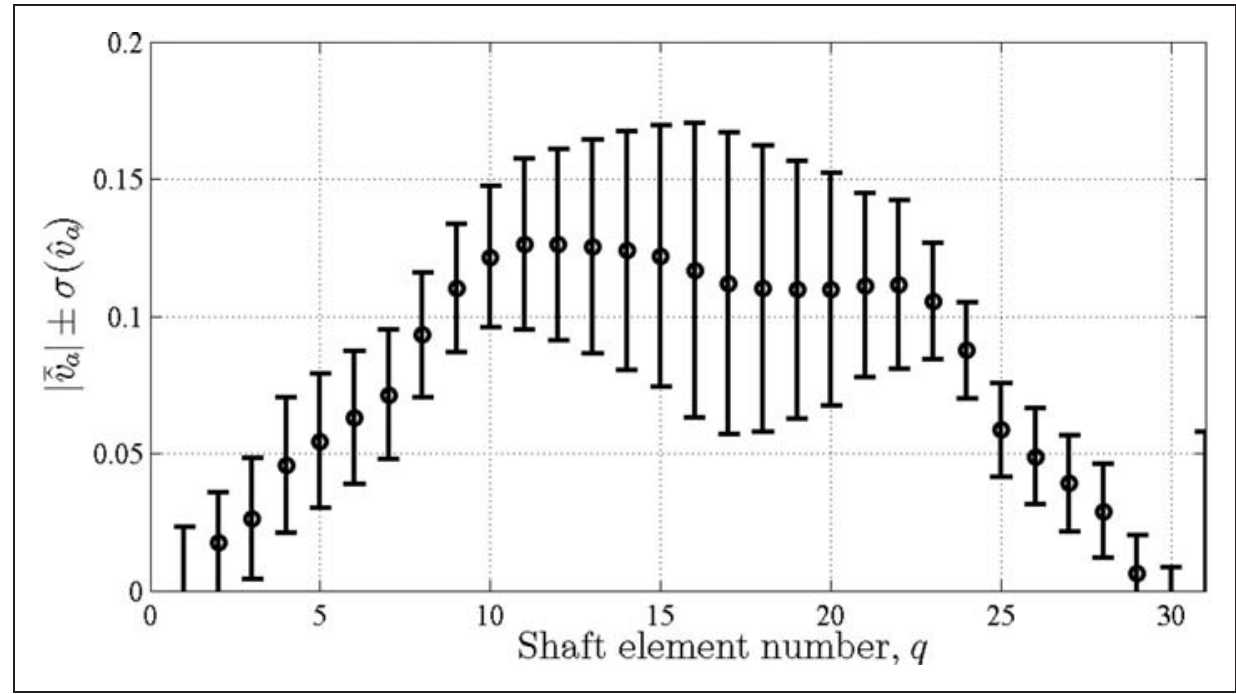

Figure 15. Absolute mean values $\hat{v}_{a}$ and standard deviations $\sigma\left(\hat{v}_{a}\right)$ fer different locations $q$ of vector $K_{f}$ within vector $K_{a}$.

To investigate the nature of the crack indicators further, we focused on their changes in time. As can be seen in Figures 11, 12 and 13 they oscillate in some way. A closer look at these oscillations is presented in Figures 16a and 17a. Fourier transforms for these oscillations are presented in Figures 16b and 17b.

For the rotor without the crack, the spectrum consists of only one synchronous component, $\omega_{1}=27 \mathrm{~Hz}$. However, for the rotor containing $40 \%$ crack, the spectrum of $\hat{x}_{a}$ consists of several components, $\omega_{1}=27 \mathrm{~Hz}$, $\omega_{2}=54 \mathrm{~Hz}, \omega_{3}=81 \mathrm{~Hz}, \omega_{4}=408 \mathrm{~Hz}$ and so on (the rest are not presented here), being integer multiples of the rotor speed, providing well-known evidence of a crack (Mani et al., 2006; Sawicki et al., 2009). Thus, not only can the estimates $\hat{x}_{a}, \hat{v}_{a}$ of the additional states themselves be used as crack indicators, but so can their Fourier spectra.

\section{Conclusions}

A new rotor crack detection method based on the control theory has been developed. Rotor crack indicators in the form of the estimates of two auxiliary state variables introduced into the FE model of the 


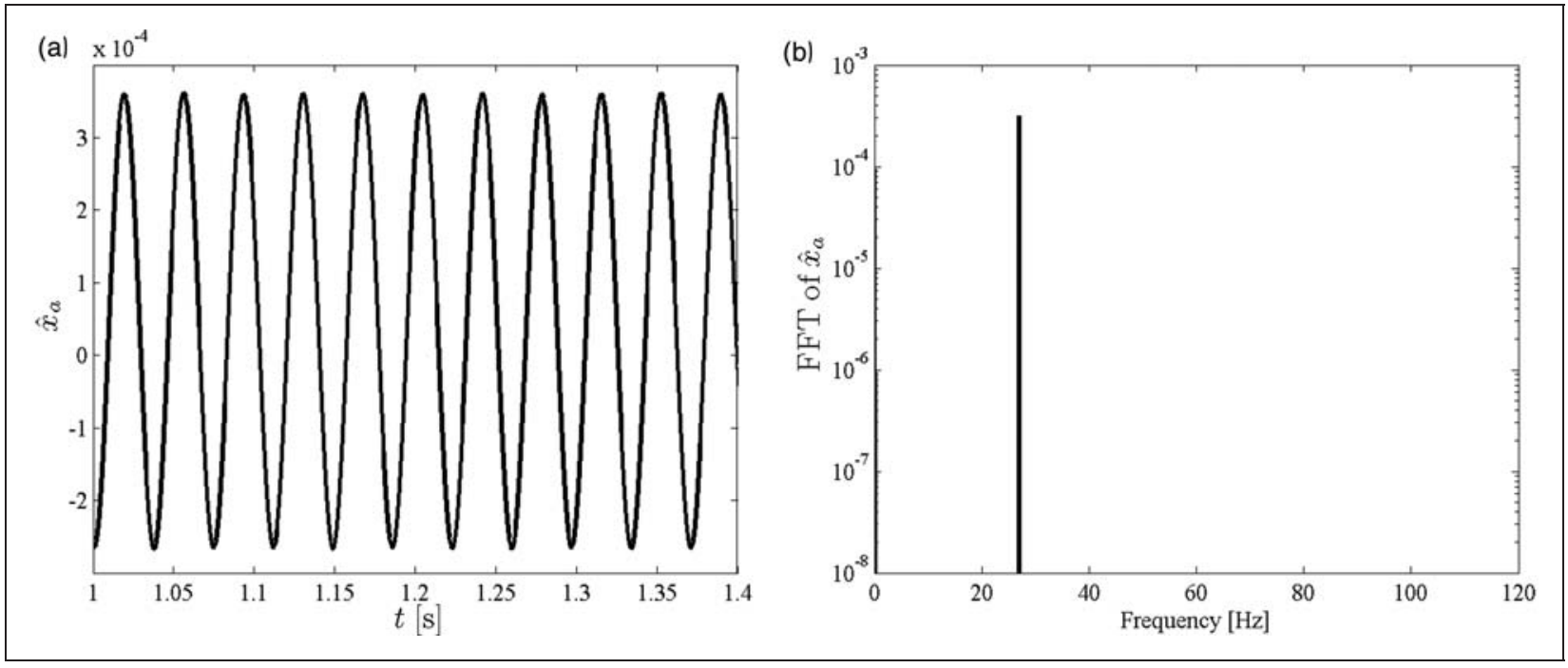

Figure 16. (a) Crack indicator $\hat{x}_{a}$ time response and (b) fast Fourier transform (FFT); no crack; $m_{a}=4, k_{a}=5.12 \times 40^{4}, k_{f y}=1$, $k_{f x}=4, q=46$.

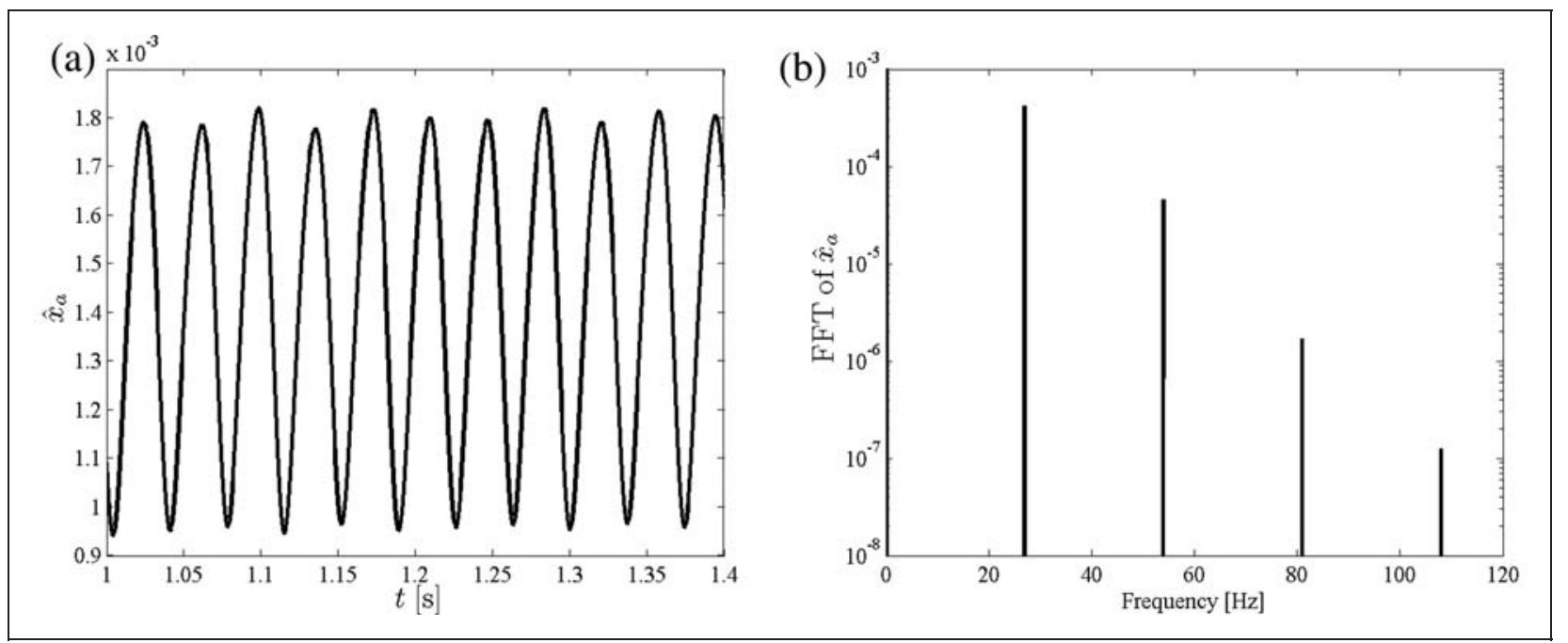

Figure 17. (a) Crack indicator $\hat{x}_{a}$ time response and (b) fast Fourier transform (FFT); $40 \%$ crack; $m_{a}=4, k_{a}=5.12 \times 40^{4}, k_{f y}=1$, $k_{f x}=4, q=46$.

rotor-bearing system have been proposed. These additional states have been introduced in terms of a simple one-degree-of-freedom oscillating system. The analysis of the properties of such a combined model of the rotor-bearing system, as well as the numerical calculation results, have shown that the estimates of these auxiliary state variables give very strong symptoms of crack presence. Not only time estimates, but also their Fourier transforms can be used as very good crack indicators. The presented method will be further analyzed and experimentally studied based on the presented crack detection rotor test rig in future work.

\section{References}

Bachschmid N, Pennacchi P, Tanzi E and Vania A (2000) Identification of transverse crack position and depth in rotor systems. Meccanica 35: 563-582.

Bently DE and Muszynska A (1986) Detection of rotor cracks, in Proceedings of Texas A\&M University 15th Turbomachinery Symposium and Short Courses, November, Corpus Christi, TX, pp. 129-139.

Darpe AK, Gupta K and Chawla A (2004) Coupled bending, longitudinal and torsional vibrations of a cracked rotor. Journal of Sound and Vibration 269: 33-60.

Dimarogonas AD and Paipetis SA (1983) Analytical Methods in Rotor Dynamics. London: Applied Science Publishers. 
Friswell MI and Penny JET (2002) Crack modeling for structural health monitoring. Structural Health Monitoring: An International Journal 1(2): 139-148.

Gasch R (1993) A survey of the dynamic behavior of a simple rotating shaft with a transverse crack. Journal of Sound and Vibration 160(2): 313-332.

Guo D and Peng ZK (2007) Vibration analysis of a cracked rotor using Hilbert-Huang transform. Mechanical Systems and Signal Processing 21: 3030-3041.

He Y, Guo D and Chu F (2001) Using genetic algorithms to detect and configure shaft crack for rotor-bearing system. Computer Methods in Applied Mechanics and Engineering 190: 5895-5906.

Ishida Y and Inoue T (2006) Detection of a rotor crack using a harmonic excitation and nonlinear vibration analysis. ASME Journal of Vibration and Acoustics 128: 741-749.

Isermann R (2005) Model-based fault detection and diagnosis - status and applications. Annual Reviews in Control 29: 71-85.

Loparo KA, Adams ML, Lin W, Abdel-Magied MF and Afshari N (2000) Fault detection and diagnosis of rotating machinery. IEEE Transactions of Industrial Electronics 47(5): 1005-1014.

Mani G, Quinn DD and Kasarda M (2006) Active health monitoring in a rotating cracked shaft using active magnetic bearings as force actuators. Journal of Sound and Vibration 294: 454-465.

Mayes IW and Davies WGR (1984) Analysis of the response of a multi-rotor-bearing system containing a transverse crack in a rotor. Journal of Vibration, Acoustics, Stress and Reliability in Design 106: 139-145.

Müller PC, Bajkowski J and Söffker D (1994) Chaotic motions and fault detection in a cracked rotor. Nonlinear Dynamics 5: 233-254.

Nelson HD (1980) A finite rotating shaft element using Timoshenko beam theory. ASME Journal of Mechanical Design 102: 793-803.

Ogata K (1997) Modern Control Engineering, 3rd edn. Englewood Cliffs, New Jersey: Prentice-Hall.

Papadopoulos CA and Dimarogonas AD (1987) Coupled longitudinal and bending vibrations of a rotating shaft with an open crack. Journal of Sound and Vibration 117: 81-93.

Park RW (2000) Noise and fault diagnosis using control theory. ICASE: The Institute of Control, Automation and System Engineering 2(1): 24-30.
Patton RJ and Chen J (1997) Observer-based fault detection and isolation: robustness and applications. Control Engineering Practice 5(5): 671-682.

Pennacchi P, Bachschmid N and Vania A (2006) A modelbased identification method of transverse cracks in rotating shafts suitable for industrial machines. Mechanical Systems and Signal Processing 20: 2112-2147.

Preumont A (2008) Active Control of Structures. London: Wiley.

Saavedra PN and Cuitino LA (2002) Vibration analysis of rotor for crack identification. Journal of Vibration and Control 8(1): 51-67.

Sawicki JT, Friswell MI, Pesch AH and Wroblewski A (2008) Condition monitoring of rotor using active magnetic actuator, in Proceedings of ASME Turbo Expo 2008: Power for Land, Sea and Air, June, Berlin, Germany, pp. 9-13.

Sawicki JT, Sen AK and Litak G (2009) Multiresolution wavelet analysis of the dynamics of a cracked rotor. International Journal of Rotating Machinery. doi:10.1155/ 2009/265198.

Sawicki JT, Wu X, Baaklini G and Gyekenyesi AL (2003) Vibration-based crack diagnosis in rotating shafts during acceleration through resonance, in Proceedings of SPIE 10th Annual International Symposium on Smart Structures and Materials, San Diego, CA.

Seibold S and Weinert K (1996) A time domain method for the localization of cracks in rotors. Journal of Sound and Vibration 195(1): 57-73.

Sekhar AS (2004) Crack identification in a rotor system: a model-based approach. Journal of Sound and Vibration 270: 887-902.

Sinou J-J and Lees AW (2005) The influence of cracks in rotating shafts. Journal of Sound and Vibration 285: 1015-1037.

Söffker D, Bajkowski J and Müller PC (1993) Detection of cracks in turborotors - a new observer-based method. ASME Journal of Dynamic Systems, Measurement and Control 3: 518-524.

Xiang J, Zhong Y, Chen X and He Z (2008) Crack detection in a shaft by combination of wavelet-based elements and genetic algorithm. International Journal of Solids and Structures 45: 4782-4795.

Xie YM (1996) An assessment of time integration schemes for non-linear dynamic equations. Journal of Sound and Vibration 192(1): 321-331. 